\title{
Power Analysis on Same Filter Different Sources for Selection of Spectral Filters in Optical Demultiplexer
}

\author{
Mohammad Syuhaimi Ab-Rahman, Hadiguna and Latifah Sarah Supian \\ Department of Electrical and Electronics, Spectrum Technology Research Group (SPECTECH), \\ Faculty of Engineering and Built Environmental, \\ University Kebangsaan Malaysia, 43600 UKM Bangi, Selangor, Malaysia
}

Received 2012-08-06, Revised 2013-06-17; Accepted 2013-07-02

\begin{abstract}
The cost effective demultiplexer based on POF is reported in this study. The demultiplexer is fabricated by combining optical splitter with the suitable filter for wavelength selection. Therefore this study we aims to evaluate the effectiveness of selected color filter film for demultiplexer construction where only signals with certain wavelengths are allowed to pass through the demultiplexer while the other signal that carrying different wavelengths are absorbed. Power reduction analysis has been carried out for special filter used in the configuration. Characterization testing and analysis is done based on recorded data. Demultiplexer components are categorized by Same Filter Different Sources (SFDS) scheme and then performance of transmitted signal by the presence of interference is studied. Demultiplexer developed is effective in short range communication, using low cost components and simple techniques. Communication range for short range communication is maximized. Only signals with certain wavelengths are allowed to pass through the multiplexer. The rest are absorbed. Several measures are made to ensure efficiency, such as testing and measurement on parameters contributing to loss. Best filters are selected based on their ability to block certain wavelengths while letting others or pass through efficiently.
\end{abstract}

Keywords: Plastic Optical Fiber, Demultiplexer, Spectral, Filter, Output Power

\section{INTRODUCTION}

Donald B. Check, a director opt-electronics research from corning incorporated is quoted as he said that " $\mathrm{A}$ global revolution is in progress. Our method of handling information is changing from electrons and electronics to light beams and photonics. These changes are occurring more rapidly than at any other time in the history of telecommunications" (Shtainhart et al., 1999). The statement made by him is realistic because in Malaysia, the trend of optical fiber communication has begun spreading in data transmission network which replacing copper-wire communication system due to high speed broadband service can be achieved via fiber optic cable-
Uni-fi ${ }^{\mathrm{TM}}$ has been firstly introduced in key "high economic impact areas" of the country. However, In future, the implementation of Uni-fi ${ }^{\mathrm{TM}}$ will follow new trend and will revolutionize in small-world communication especially in Malaysia. Polymer Optical Fiber (POF) data communications are possible offering wide advantages over its counterpart, copper wire and glass fiber. Among benefits offered are cost-effective in areas from raw material cost, processing, connection costs, high coupling efficiency with Light Emitting Diode (LED) (emitting area around $300 \mu \mathrm{m}^{2}$ ), Electromagnetic Interference (EMI) immunity, no grounding necessary, resistance to heat and narrow bending radius (Cisco System, 2000; Gupta and Khurana, 2010).

Corresponding Author: Mohammad Syuhaimi Ab-Rahman, Department of Electrical and Electronics,

Spectrum Technology Research Group (SPECTECH), Faculty of Engineering and Built Environmental, University Kebangsaan Malaysia, 43600 UKM Bangi, Selangor, Malaysia 
Today, the whole world of telecommunications and information communities is facing more and more serious challenges, namely on one side of transmitted multimedia-rich data are exploding at an astonishing speed and on the other side the total energy consumption by the communication and networking devices and the relevant global $\mathrm{CO}_{2}$ emission are increasing terribly. It has been pointed out that currently $3 \%$ of the world-wide energy is consumed by the Information and Communications Technology (ICT) infrastructure that causes about $2 \%$ of the world-wide $\mathrm{CO}_{2}$ emissions, which is comparable to the world-wide $\mathrm{CO}_{2}$ emissions by airplanes or one quarter of the world-wide $\mathrm{CO}_{2}$ emissions by cars (Cisco System, 2000).

Silica or Glass Optical Fiber (GOF) recently has become a 'trendsetter' in Fiber To The Home (FTTH) technology to provide a high capacity communication data which be transmitted to a user from central office. But, not much people-or even Network Service Provider (NSP) itself- are alerts about the safety concern of the technology, especially for user. Lasers, as the best transmission media utilize together with GOF could be very dangerous once the leakage occurs from the body structure of the GOF itself. A very high intensity ray of light released by fiber can possibly burn a human retinas and lead to a permanent blind.

Combination between POF which very suitable to a LED technology can be seen as the best solution to provide a communication data services which is much safer and not to mention about the lowest price we can get for the capital and fabrication cost. POF links are increasingly popular for applications such as computer or peripheral connections, control and monitoring, board interconnects and even domestic hi-fi systems. Unlike GOF, POF remains flexible while having a large diameter core and high numerical aperture (Gupta and Khurana, 2010) lead to a high capacity that they can bring along the fiber.

Recent communication system over POF desires increasingly more bandwidth and therefore the WDM system is the solution that allows the transmission of information over more than just a single wavelength and thus greatly increases the POF's bandwidth. WDM is a technique that multiple signals are carried together as separate wavelengths of light in a multiplexed signal. Wavelength Division Multiplexer is the first passive device required in WDM-POF system and it functions to combine optical signals from multiple different singlewavelength end devices onto a single fiber.

In WDM-POF system, each transmitter with different lights color carries single information. For example, red light with $650 \mathrm{~nm}$ wavelength modulated with Ethernet signal while blue, green and yellow lights carry image information, Radio Frequency (RF) and television signal, respectively (Imoto et al., 1987). Conceptually, the same device can also perform the reverse process with the same WDM techniques, in which the data stream with multiple wavelengths decomposed into multiple single wavelength data streams. The reverse process is called as de-multiplexing. Theoretically, POF splitter has similar function, operates to couple or combine several optical data pulses as a single coupled signal. Hence, the development of wavelength division multiplexer based on POF splitter is possible. A low-cost solution for POF-WDM system application will be presented.

Many fabrication technologies were reported for the realization of POF splitter/coupler; twisting and fusion, side polishing, chemical etching, cutting and gluing, thermal deformation, molding, biconical body and reflective body (IEC, 2007). Among these methods, the twisting and fusion or well known as Fused Biconical Taper (FBT) technology is the most predominantly practiced fabrication technique to produce a commercial splitter/coupler since the fused tapered fiber structure appears to be the most promising device. Refer to Fig. 1 (Imoto et al., 1987) has described several advantages of the fusion technique for Glass Optical Fiber (GOF) namely low loss, small loss deviation, large port number, coupling ratio independent of wavelength, coupling ratio independent of incident excitation, easy and efficient coupling to fibers and high stability against temperature variation, humidity, mechanical vibration and impact.

Ab-Rahman et al. (2011c) has demonstrate for the first time the POF devices can be fabricated by the skillful hand. The temperature, stress and splitting technique are the most important parameters to fabricate low loss device. With some modification the device can be used for the extended function such as demultiplexer which is fabricated from uniformity optical splitter (AbRahman et al., 2011a). In WDM-POF system, many transmitters with different lights color to carry single information. For example, red light with $650 \mathrm{~nm}$ wavelength modulated with Ethernet signal while blue, green and yellow lights carry image information, Radio Frequency (RF) and television signal, respectively (AbRahman et al., 2012c). Wavelength Division Multiplexer is the first passive device required in WDM-POF system and it functions to combines optical signals from multiple different single-wavelength end devices onto a single fiber (Ab-Rahman et al., 2012b). Conceptually, the same device can also perform the reverse process with the same WDM techniques, in which the data stream with multiple wavelengths decomposed into multiple single wavelength data streams. 


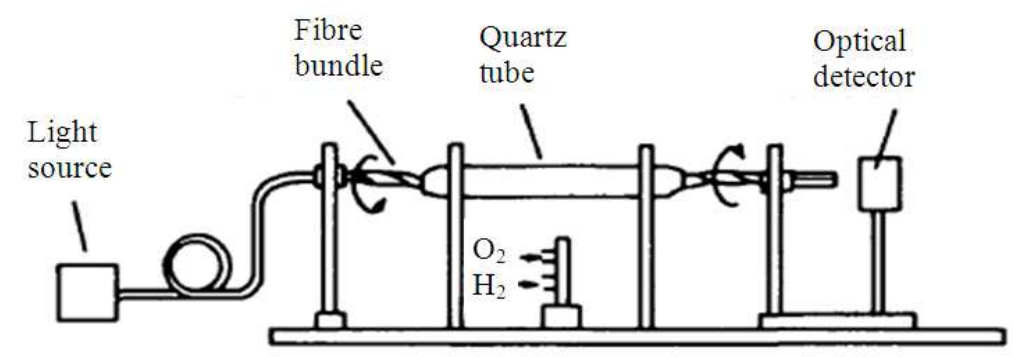

(a)

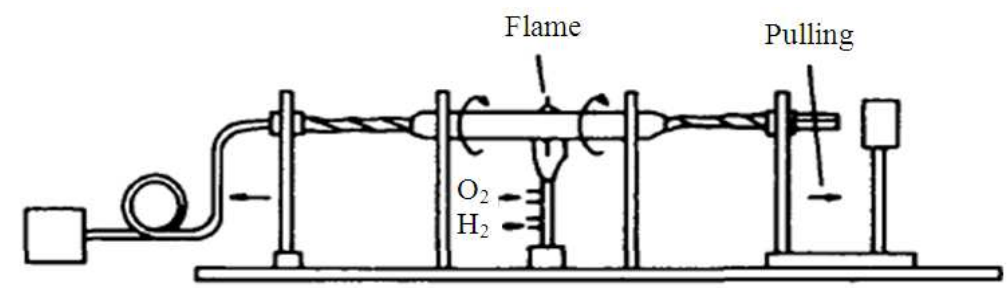

(b)

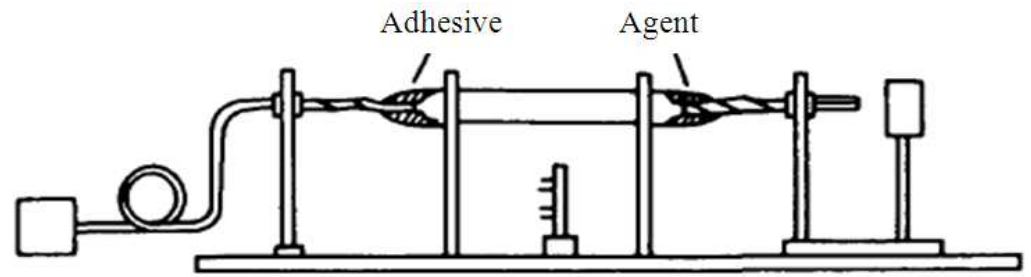

(c)

Fig. 1. Conventional FBT method for the fabrication of fused splitting/coupling device for GOF (Source: Imoto et al., 1987)

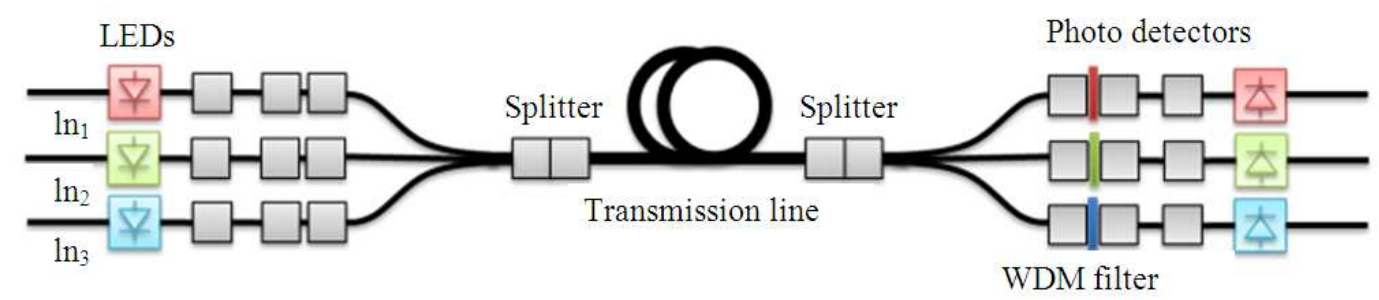

Fig. 2. Eco-friendly-WDM-POF network architecture using $1 \times 3$ splitter and color filters. The splitter can also be used as multiplexer and the splitter-filter combination work as a demultiplexer

The reverse process is called as de-multiplexing. Conceptually, POF splitter has similar function, operates to couple or combine several optical data pulse as a single coupled signal. Hence, the development of wavelength division multiplexer based on POF splitter is possible. A low-cost solution for POF-WDM system application will be presented.

A novel fused POF splitter will fabricated by a fusion technique as reported by Ab-Rahman et al. (2011b), as an effective transmission media to split and recombine a number of different wavelengths which represents different signals. Three different wavelengths will be fully utilized to transmit three different sources of systems; LAN connection Network, radio (audio) and video transmission system. Red LED which in $665 \mathrm{~nm}$ wavelength capable to download and upload data through Ethernet cable while green LED in $520 \mathrm{~nm}$ wavelength can transmit a video image generated from 
DVD player or CCTV system and blue LED with 470 $\mathrm{nm}$ wavelength represents an audio transmission system inside the house (Ab-Rahman et al., 2012a). Special filter will be placed between the splitter and receiver-end to make sure the entire WDM system can select a single signal as desired. For the filter design which able to eliminate unwanted signal and select the wavelength of the system as desired as shown in Fig. 2. Some parameters, such as optical output power, power losses, optical noise to ratio and crosstalk of the devices can be observed and not mentioning about the effect of filter placement and the efficiency of the WDM-POF system itself.

\subsection{Objectives}

The objectives of characterization and analysis testing are to develop de multiplexer component and optical splitter that provide optimal results when applied to the data transmission systems. Next is to evaluate the effectiveness of selected color filter film for demultiplexer construction where only signals with certain wavelengths are allowed to pass through the demultiplexer while the other signals carrying different wavelengths are absorbed.

Characterization testing and analysis are done based on recorded data. The developed demultiplexer components is Same Filter Different Sources (SFDS). From the final analysis, the outcomes of the research are:

- The developed demultiplexer is effective to be applied in short range communication system by using low-cost components and implementation techniques that are not too complicated

- Maximum distance can be achieved for short range communications system by using the components developed

- Signals that carry different wavelengths are absorbed or prevented from being transmit through the developed de multiplexer with filter film. Only signals with certain wavelengths are allowed to pass through the de multiplexer

- Signal transmitted through optical fiber and optical splitter by using LED light source is tested for the maximum distance that can be achieved for the purpose of data transmission. Parameters that have contributed to the loss of signals (which are carried by the components developed) are tested and measured. It is done to ensure the interference that exists in data transmission system does not affect the total emitted signal or reduced the performance of system that was developed in order to maintain the performance of signal transmission efficiency

\subsection{Same Filter Different Sources (SFDS)}

Based one experiments, the red LED signal which has been injected in to optical fiber that attached to filter film has a decreases value of insertion loss and output power. The same situation occurs when fiber is injected with blue and green LED light source. For analytical characterization of same filter film that has been injected with different sources of LEDs which are red, blue and green; the filter sample A (\#4690) is used as a red film filter and injected with red, blue and green LED. Based on the collected data, a filter film of \#4690 that has been injected with red LED in dicatesa small insertion loss which is $0.8 \mathrm{dBm}$. The recorded output power also shows the power reduction after the filter film is installed, which is $5.3 \mu \mathrm{W}$. Transmitted signal loss is due to the use of epoxy-resin adhesive that spread on to the surface of optical fiber when filter film is attached. The spreading glue prevents a continuous wavelength signals from being transmitted through the filter film.

However, the results of different data are observed when blue and green LED source light are injected through\#4690 red filter film. There is only a little of insertion loss for blue and green LED signals that transmitted through there filter film. This is because, \#4690 red filter films cannot prevent most of blue and green wavelengths from passing through the film. Therefore, blue and green wavelengths can be transmitted through red filter film. It shows the \#4690 red filter film cannot be used as a demultiplexer because of the film's characteristic, where it cannot absorb or prevent unknown wavelengths to pass through. Based on the recorded output power, the efficiency of filter film is evaluated. In addition, from the graph that has been plotted, the effectiveness of \#4690 red filter films does not show an outstanding result in order to develop a demultiplexer.

Figure 3 shows the percentage rate of decline in output power of \#4690 filter films in which has negative percentage value shows the increasing rate in measured output power. Filter film allows LED light source with different wavelengths pass through. Based on data analysis, this filter film is not suitable to be used as a demultiplexer because it cannot prevent other light source from passing through.\#318 red filters film is used as a sample and testing process as previously done. Insertion loss rate of the reading shows a loss of $0.2 \mathrm{dBm}$ after \#318 red filter films has been attached to optical fibers as depicted in Fig. 4. A factor contributing to small signal loss is due to the use of epoxy-resin adhesive that spread on to the surface of optical fibers. Adhesive that spread on surface of optical fibers has prevented the transmission of emission wavelength directly through filter film. 
The reduction in output power (uW) of red, green and blue LED that injected through \#4690 red filter films

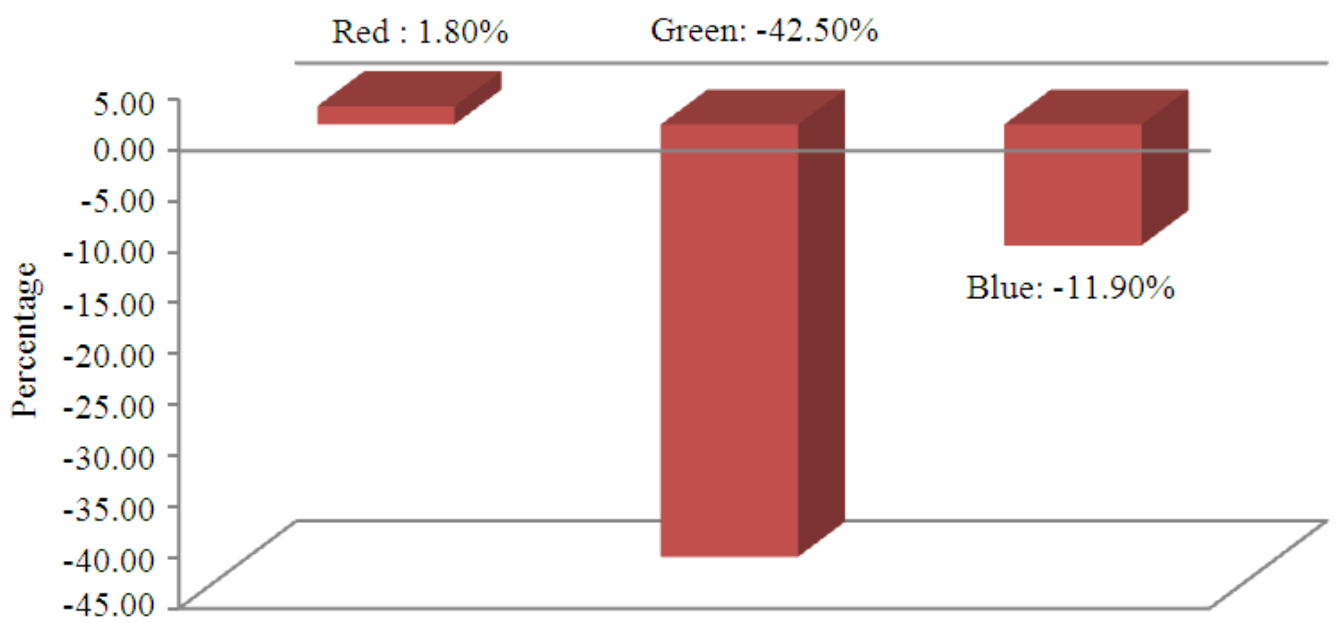

Blue, green, red LED

Fig. 3. Percentage of reduction in output power (efficiency) for \#4690 red filter films when light sources (red, green and blue LED) are injected

The increase in insertion loss rates $(\mathrm{dBm})$ of red, blue and green LED that injected to \#318 red filter film

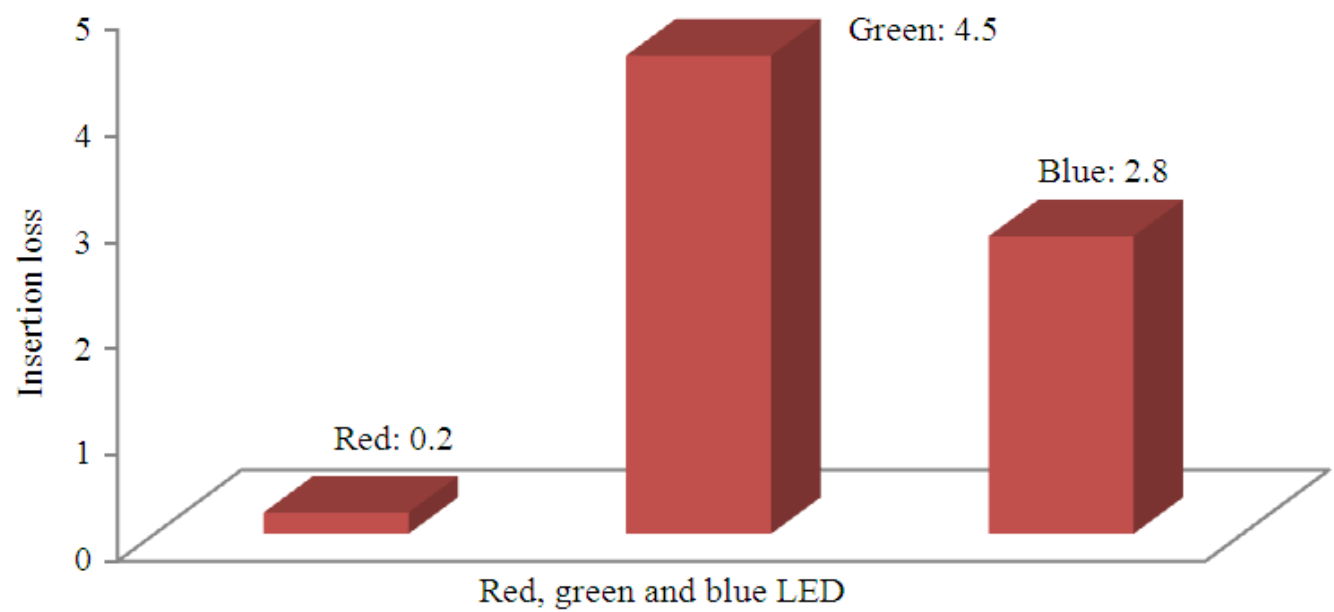

Fig. 4. The increase in insertion loss $(\mathrm{dBm})$ of red, blue and green LED when injected through optical fiber and \#318 red filter films

The same situation is found when the light source of blue and green LED is injected into optical fiber through \#318 red filter films. In section loss rates increased when the blue and green LED has emitted.

Effectiveness has evaluated using the reduction in percentage data of output power that has been recorded. Referring to Fig. 5, the signal wave length of red LED shows a small reduction in output power. While the percentage of output power for signal wave length of blue and green LED shows a large reduction in rates. In other words, most of the wave length that carrying blue and green light sources absorbed or prevented from transmit through\#318 red filter films. Only signal wavelength that carried red LED allowed to go through the filter film. Based on the analysis, the \#318 red filter films could be an effective demultiplexer. 
Mohammad Syuhaimi Ab-Rahman et al. / Journal of Computer Science 9 (7): 866-874, 2013

The reduction in output power $(\mathrm{uW})$ of red, green and blue LED that injected through $\# 318$ red filter films

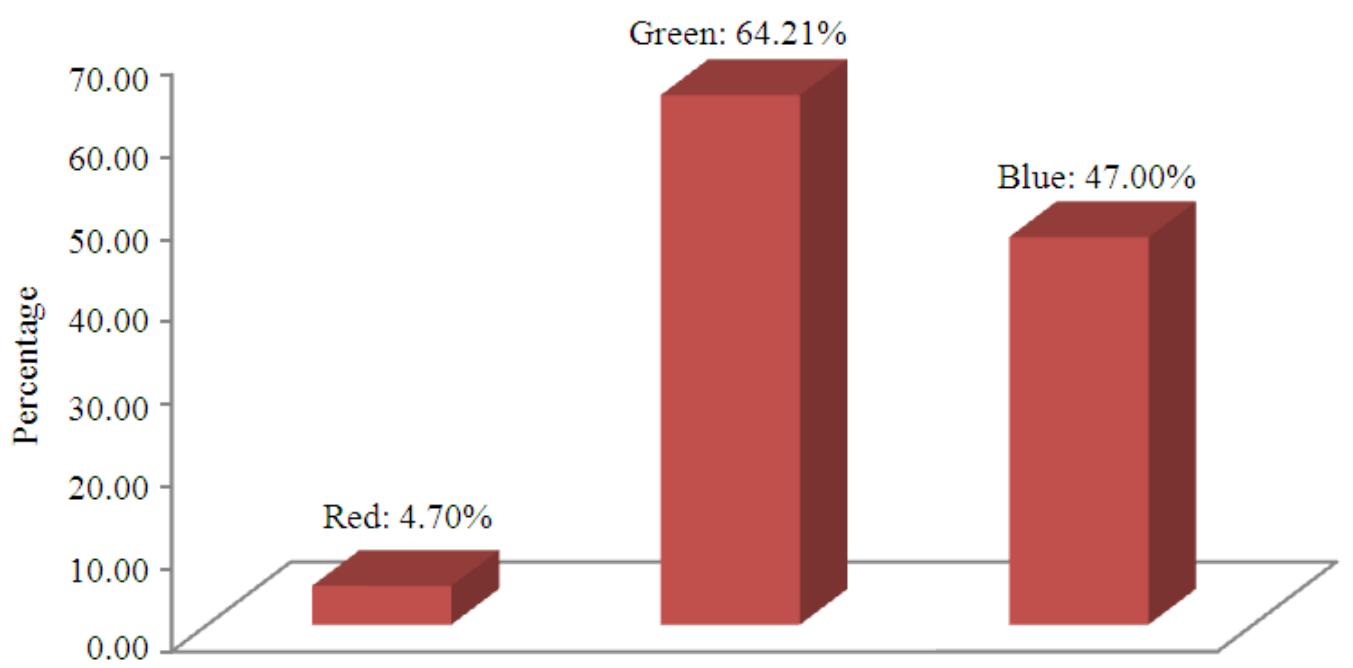

Red, green and blue LED

Fig. 5. Percentage of output power rate (efficiency) for \#318 red filter films when light sources (red, green and blue LED) are injected

The reduction in output power $(\mathrm{uW})$ of red, green and blue LED that injected through $\# 62$ blue filter films

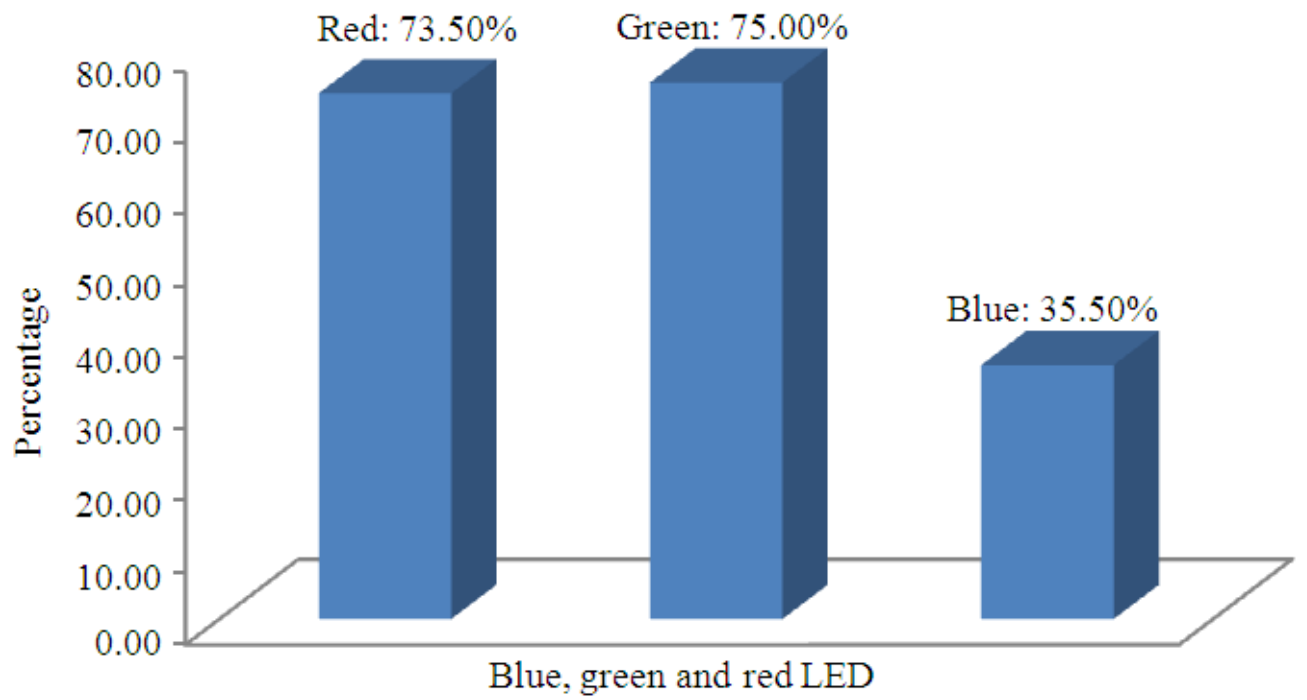

Fig. 6. Percentage of output power rate (efficiency) for \#62 blue filter films when light sources (red, green and blue LED) are injected

Characterization analysis also has been performed for blue filter film where it has injected with red, blue and green LED. \#62blue samples shows the least reduction percentage of the output power where more than $60 \%$ of blue LED light can go through the film. This film also prevents other light sources to go through it. Bar graph in Fig. 6 shows the reduction percentage in output power (efficiency) for blue\#62 films. 
The increase in insertion loss rates $(\mathrm{dBm})$ of red, blue and green LED that injected to \#62 blue filter film

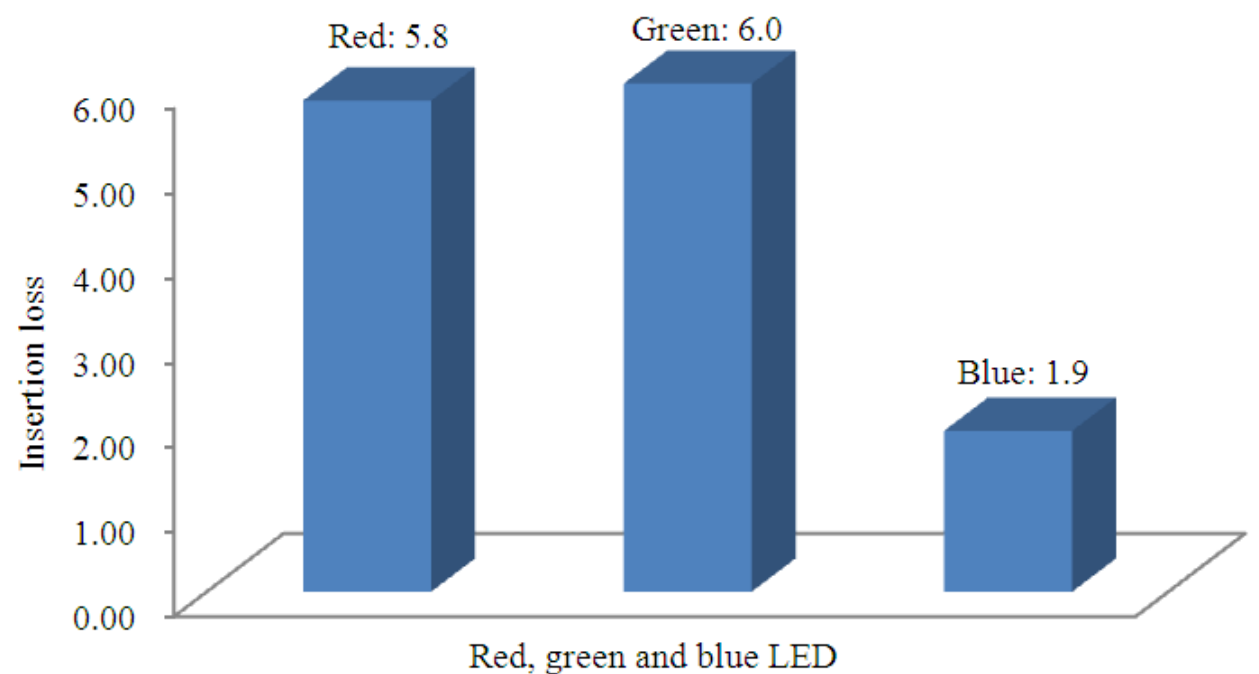

Fig. 7. The increase in insertion loss $(\mathrm{dBm})$ of red, blue and green LED when injected through optical fiber and \#62 blue filter films

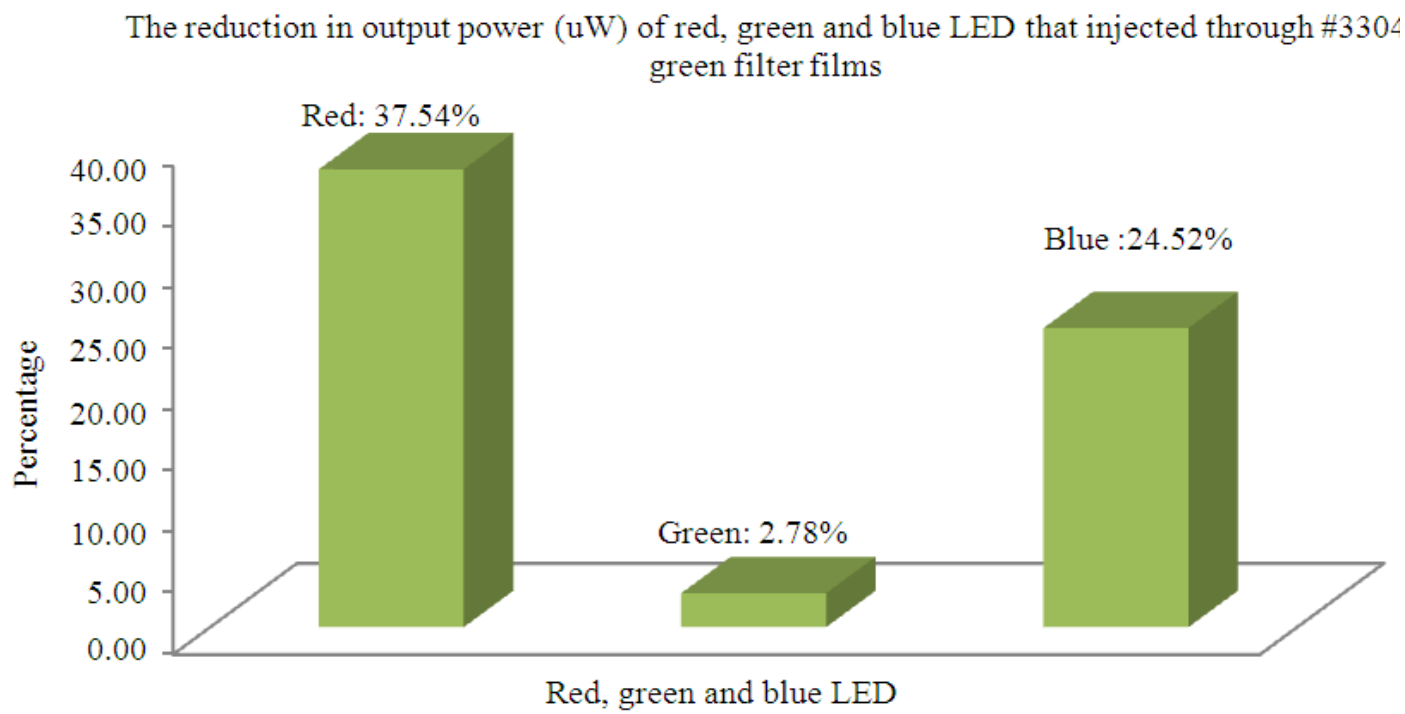

Fig. 8. Percentage of output power rate (efficiency) for \#3304 green filter films when light sources (red, green and blue LED) are injected

Only signal wavelength that carried blue LED light sources is allowed to go through the filter film. Based on analysis, the \#62Blue filter films could be an effective demultiplexer.

Figure 7 shows the rate of insertion loss $(\mathrm{dBm})$ for \#62blue filter film when light sources of red, blue and green LED is injected through the optical fiber and filter film. In sertion loss is high for light sources of red and green LED because blue filter film sabsorbor prevent most of red and green wave length stopass through it. Insertion loss rate for light source of \#62 blue filter films is small because blue wavelength can go through filter film without experiencing high loss rates. Further characterization analyses are conducted for green filter film that has injected with red, blue and green LED. 


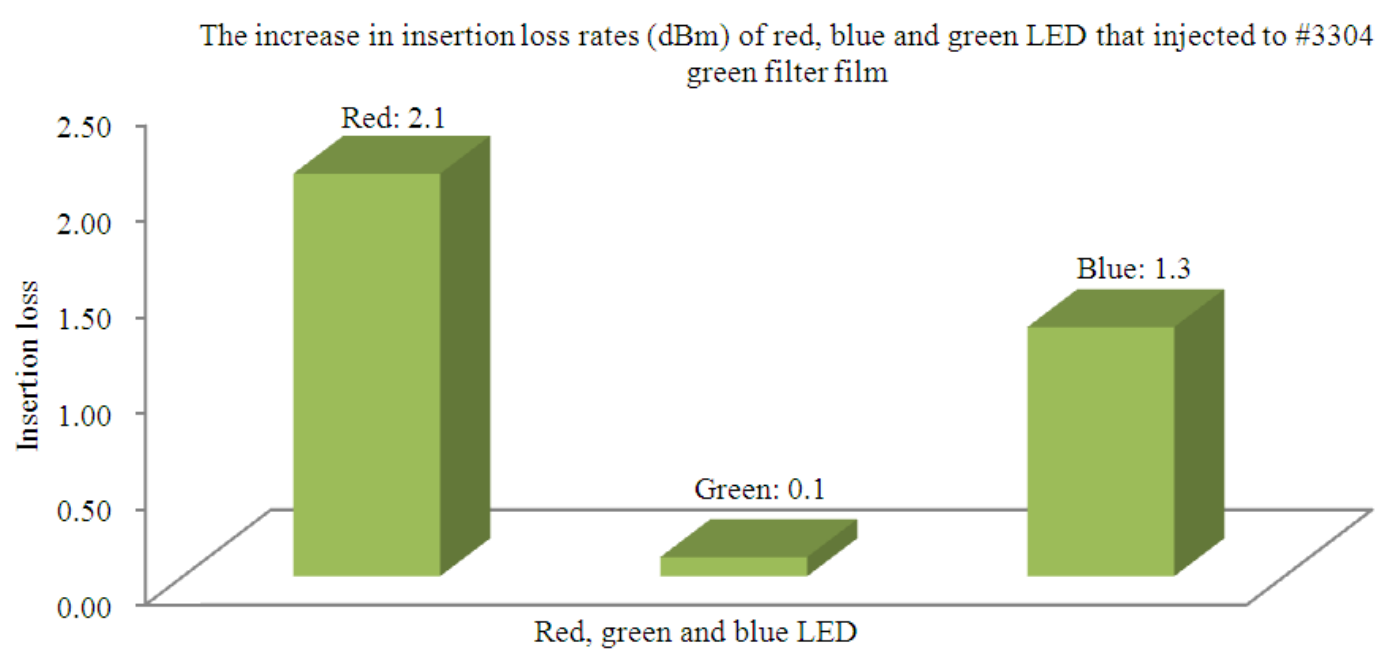

Fig. 9. The increase in insertion loss $(\mathrm{dBm})$ of red, blue and green LED when injected through optical fiber and \#3304 green filter films

The reduction in output power $(\mathrm{uW})$ of red, green and blue LED that injected through \#3304 green filter films

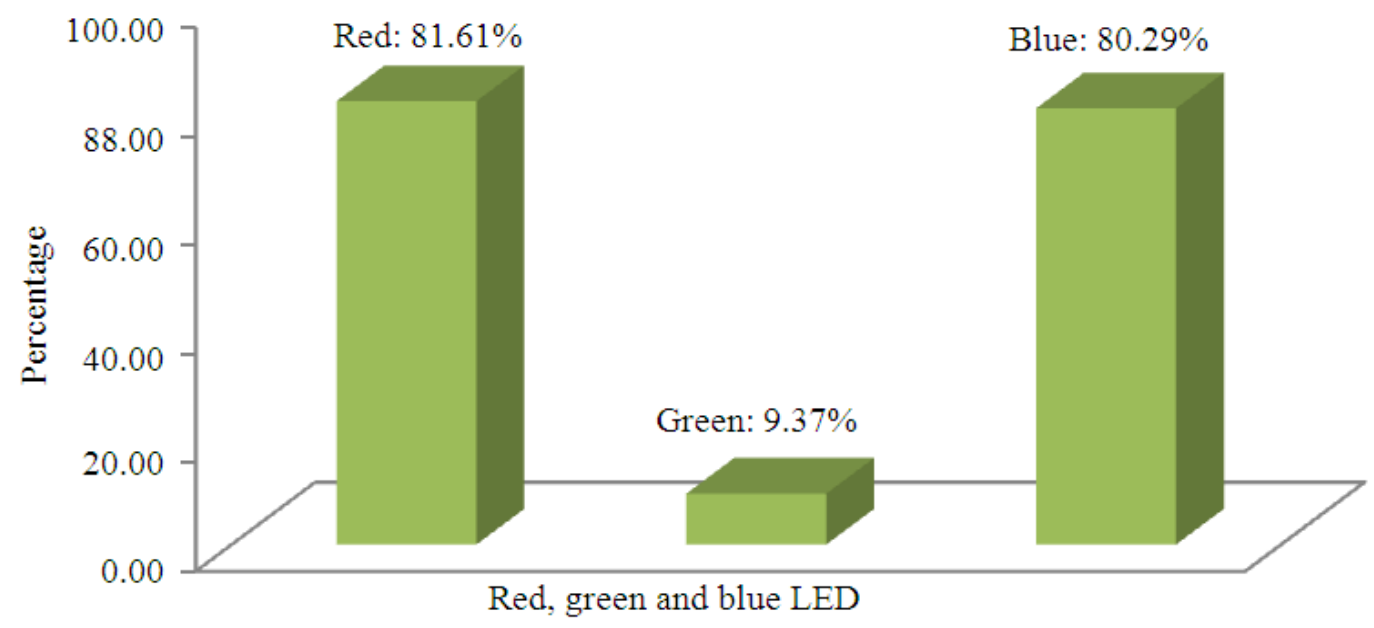

Fig. 10. Percentage of output power rate (efficiency) for \#3304 green filter films when light sources (red, green and blue LED) are injected

\#3304green samples showed that; the least reduction of percentage in output power where almost $97 \%$ green LED light can go through the film and absorbor blocks other light sources with different wave length stopass through. Figure 8 shows the reduction of percentage in output power (efficiency) for \#3304 green films. Only signal wave length that carried by green LED light source is allowed o go through the film. Based on analysis, the \#3304green filter films could be an effective demultiplexer.

Figure 9 shows the insertion loss rate $(\mathrm{dBm})$ for \#3304 green filter film where red, blue and green LED light source has injected through optical fiber and film filter. Insertion loss is high for red and blue LED light source. This is because, the film absorbs green filter or block most of red and blue wave length stop as through it. The insertion loss of green filter film for \#3304 LED light source is so small because blue wave lengths can go through the filter film without experiencing high los rates. Characterization analyses also being conducted for \#86 green filter films that has injected with red, blue and green LED where it showed that; the least reduction of percentage in output power. Almost $90 \%$ green LED light can go through the film and absorbor block other 
light sources with different wave length stop as through. Figure 10 shows the reduction of percentage in output power (efficiency) for \#86 green films. Only signal wave length that carried by green LED light source is allowed to go through the film. Based on analysis, the \#86green filter films also could be an effective demultiplexer as well as \#3304 samples.

\section{CONCLUSION}

Demultiplexer components are categorized by SFDS scheme and the performance of transmitted signal by the presence of interference is studied Characterization testing and analysis is done based on recorded data where three filters has been selected with has different colour (red, blue and green). Only signals with certain wavelengths are allowed to pass through the multiplexer. The rest are absorbed. Several measures are made to ensure efficiency, such as testing and measurement on parameters contributing to loss. Best filters are selected based on their ability to block certain wavelengths while letting others or pass through efficiently.

\section{REFERENCES}

Ab-Rahman, M.S., H. Guna and K. Jumari, 2011c. Low Cost Fused Tapered $\left(\mathrm{LFT}^{\mathrm{TM}}\right)$ splitter for multichannel WDM-POF network design. Australian J. Basic Applied Sci., 5: 156-164.

Ab-Rahman, M.S., Hadiguna and M.H. Harun, 2011a. The importance of uniformity to demultiplexer device fabrication for POF based small world communication. Adv. Natural Applied Sci., 5: 166-170.
Ab-Rahman, M.S., L.S. Supian, H. Guna, M.H. Harun and K. Jumari, 2012b. Performance of thin-film as wavelength filter in WDM-POF network. Am. J. Applied Sci., 9: 180-185. DOI: 10.3844/ajassp.2012.180.185

Ab-Rahman, M.S., L.S. Supian, H. Guna, M.H. Harun and K. Jumari, 2012c. Low-loss hand-made demultiplexer using transparent color filter in wdm-pof network for short-haul communication system. J. Comput. Sci., 8: 494-498. DOI: 10.3844/jcssp.2012.494.498

Ab-Rahman, M.S., M.H. Harun, M.H. Guna and K. Jumari, 2011b. Second generation of Low-cost Fused Tapered (LFT) splitters based on POF technology. J. Sci. Indus. Res., 70: 435-442.

Ab-Rahman, M.S., N.N.S. Mohammad, M.H Harun and L.S. Sufian, 2012a. Customer-made $3 \times 3$ polymer optical fiber splitter based on green technology using fusion technique. J. Comput. Sci., 8: 591-598. DOI: $10.3844 /$ jcssp.2012.591.598

Cisco System, 2000. Inc. Introducing DWDM.

Gupta, P. and H. Khurana, 2010. Public Entrepreneurship: A dynamic strength for budding green technology. Proceedings of the 4th National Conference, Feb. 2526, Bharati Vidyapeeth's Institute of Computer Applications and Management, New Delhi.

IEC, 2007. Dense Wavelength Division Multiplexing (DWDM).

Imoto, K., M. Maeda, H. Kunugiyama and T. Shiota, 1987. New biconically tapered fiber star coupler fabricated by indirect heating method. Light Wave Technol., J., 5: 694-699. DOI: 10.1109/JLT.1987.1075559

Shtainhart, A., R. Segal and A. Tsherniak, 1999. Wavelength-Division Multiplexing (WDM). 